ASTHMA

\title{
Diagnostic accuracy of bronchodilator responsiveness in wheezy children
}

\author{
I Dundas, E Y Chan, P D Bridge, S A McKenzie
}

Thorax 2005;60:13-16. doi: 10.1136/thx.2004.029934

See end of article for authors' affiliations

.....................

Correspondence to: Dr I Dundas, Department of Paediatric Respiratory Medicine, The Royal London Hospital, Barts and The London NHS Trust, London El 1BB, UK; I.Dundas@qmul.ac.uk

Received 9 June 2004 Accepted 7 October 2004

\begin{abstract}
Background: The aim of this study was to evaluate the diagnostic accuracy of bronchodilator responsiveness for asthma in 5-10 year old children.

Methods: Spirometric measurements were made in 142 children (58 wheezers) before and after $400 \mu \mathrm{g}$ inhaled salbutamol.

Results: On a receiver operating characteristic curve, a $9 \%$ increase in predicted forced expiratory volume in 1 second was the cut off point that provided an acceptable balance of sensitivity and specificity for previous wheeze. This figure was $50 \%$ (95\% Cl 38 to 62) sensitive and $86 \%$ (95\% Cl 78 to 92 ) specific for detecting previous wheeze and multiplied the initial odds in favour of wheeze by a factor of $3.6195 \% \mathrm{Cl}$ 2.0 to 6.3 ).

Discussion: With an estimated pretest probability of wheeze of $10 \%$ in the community and $50 \%$ in a specialist clinic, the positive predictive values are $29 \%$ and $78 \%$, respectively, for a $9 \%$ change. The value of bronchodilator responsiveness testing depends on the prevalence of wheeze in the population in which it is to be used.
\end{abstract}

A s one of the hallmarks of asthma is airway lability, it may be helpful when diagnosing asthma to try to demonstrate this by measuring lung function before and after administering a bronchodilator. United States guidelines on asthma management suggest that an asthma work-up should include measurement of bronchodilator responsiveness (BDR). ${ }^{1}$ For the diagnosis of asthma in schoolchildren the British Thoracic Society ${ }^{2}$ has advised “... bronchodilator responsiveness, peak expiratory flow variability or tests of bronchial hyperreactivity may be used to confirm the diagnosis, with the same reservations as in adults". However, in this guideline BDR is not defined nor are the reservations described. A recent review of the value of lung function testing in adults suggested that many tests used for diagnosis and for assessing a known condition are not supported by high quality evidence. ${ }^{3}$

Response to a bronchodilator is characterised by an increase in forced expiratory flows (including peak expiratory flow) and volumes, and a decrease in airway resistance. ${ }^{45}$ In adults an increase in forced expiratory volume in 1 second $\left(\mathrm{FEV}_{1}\right)$ of at least $12-15 \%$ from the baseline value and an absolute change of $200 \mathrm{ml}$ is defined as a meaningful response-that is, 95th centile of the difference between measurements in a group of healthy subjects before and after bronchodilator. ${ }^{6}$ In other words, these measurements give a specificity of $95 \%$ for asthma. In a population study of 7 year old children ${ }^{7}$ an improvement of $10.2 \%$, calculated from the reported coefficient of variation of a measurement of $4.3 \%$ $\left(1.64^{*} 4.3 \%^{*} \sqrt{2}\right)$, represented the 95 th centile of the mean difference in percentage predicted $\mathrm{FEV}_{1}$ before and after bronchodilator. In practice, how does BDR testing perform as a diagnostic test in the most challenging group of children presenting to the clinician-namely, those with vague respiratory symptoms which might be explained by asthma? The aim of this study is to evaluate the diagnostic accuracy of BDR testing in children being investigated for mild intermittent wheeze at step 1 of the BTS guideline. ${ }^{2}$

\section{METHODS}

In the control or "non-wheezer" group the children were aged 5-9 years inclusive with no personal history of wheeze, difficulty in breathing or asthma; those with isolated cough were not excluded. Children were included as controls if prebronchodilator $\mathrm{FEV}_{1}$ was greater than $80 \%$ predicted for height (that is, within the normal range). ${ }^{8}$ They were recruited from east London schools and outpatient clinics. Wheezers were children of similar age with a history of at least one episode of doctor observed wheeze responding to bronchodilator treatment within the previous 3 months. These children were recruited consecutively from the hospital ambulatory department. No child was receiving medication other than bronchodilator as needed, and were at step 1 of the BTS guideline. ${ }^{9}$ Children already at step 2 and above were excluded. Children were excluded if they were given a bronchodilator within 24 hours of the test. Children with upper respiratory tract infections in the previous 3 weeks were excluded. The study took place between June 1999 and May 2003

Two clinicians and two clinical scientists fully trained in respiratory function measurements recruited and measured children for this study, adhering to locally modified American Thoracic Society standards for test performance and analysis. ${ }^{6}$ Height was measured using a Harpenden stadiometer calibrated with a 1 metre rod. Lung function measurements were made with a Vitalograph compact spirometer (Buckingham, UK) calibrated using known volumes before each measurement session. Spirometric manoeuvres were performed with the child standing and without nose clips. $\mathrm{FEV}_{1}$ was reported from the best technically acceptable flowvolume loop, where rater criteria for acceptability were a rapid rise to peak flow without coughing or interruption of expiration. ${ }^{6}$ The volume-time curve was inspected to ensure expiration was more than 1 second and forced expiration was undertaken without hesitation. We did not adopt the ATS adult criteria ${ }^{6}$ of the best sum of $\mathrm{FEV}_{1}$ and FVC because, although younger children may manage a forced maximal expiration, they frequently cannot exhale down to residual volume so that FVC cannot be reported reliably. ${ }^{10}$ Measurements were repeated 15 minutes after inhalation of $400 \mu \mathrm{g}$ salbutamol via a spacer (Volumatic, Allen \& Hanburys, UK). BDR was defined as change in percent predicted $\mathrm{FEV}_{1}{ }^{*}$ 100/baseline percent predicted $\mathrm{FEV}_{1}{ }^{11}$ 
East London and the City Research ethics committee approved the project and parents and children old enough to understand the project gave written informed consent for the study.

\section{Data analysis}

Data were inspected for normality and analysed using SPSS version 11.0.1. Comparison between groups was by unpaired $t$ testing for continuous variables and by comparison of proportions for categorical variables. Data were used to plot a receiver operator characteristic (ROC) curve to describe the sensitivity and specificity of BDR for previous wheeze. Likelihood ratios, odds and post test probabilities (positive and negative predictive values) were calculated. ${ }^{12}$

\section{RESULTS}

Technically acceptable measurements were obtained in 142 children (84 non-wheezers). Ten children (four nonwheezers) consented to the study but failed to produce technically acceptable measurements. Reasons for failure were poor effort, insufficient expiratory time (FEV $<1$ second), and expiration interrupted by coughing or glottis closure. Wheezers attended for measurements a median of 6 weeks (range 4-14) following attendance in the ambulatory unit. The two groups were of similar age and sex but wheezers were slightly shorter and younger (table 1). All continuous variables were normally distributed. Percentage predicted baseline $\mathrm{FEV}_{1}$ measurements were significantly lower in wheezers and BDR was significantly greater in wheezers than in non-wheezers (table 1 ).

From an ROC curve (fig 1), cut off points for BDR were compared (table 2). The cut off point where the sum of sensitivity and specificity was greatest was 9\% change in predicted $\mathrm{FEV}_{1}$. At this point $50 \%$ of the wheezers had at least 9.0\% improvement in $\mathrm{FEV}_{1}$ following bronchodilator compared with $14 \%$ of non-wheezers.

The positive and negative likelihood ratios were 3.6 (95\% CI 2.0 to 6.3 ) and 0.6 (95\% CI 0.4 to 0.8 ). In other words, a response to bronchodilator of at least $9.0 \%$ BDR multiplies the initial odds in favour of wheeze by a factor of 3.6 and a negative test multiplies the initial odds against wheeze by a factor of $1 / 0.6=1.7$. The area under the ROC curve (AUC) was 0.71 (95\% CI 0.62 to 0.81 ).

The prevalence of asthma/wheeze is likely to differ quite widely in different circumstances. Accordingly, the positive and negative predictive values ${ }^{12}$ of a threshold of $9.0 \%$ BDR were calculated for a range of hypothetical asthma prevalences (table 3 ).

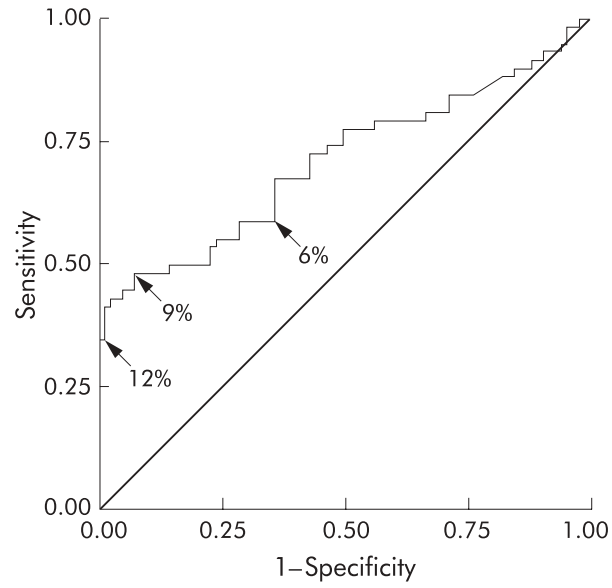

Figure 1 Sensitivity and specificity of bronchodilator responsiveness (BDR) for previous wheeze. Solid line denotes line of identity.

\section{DISCUSSION}

The aim of this prospective study was to evaluate the diagnostic accuracy of BDR as a test for asthma. We attempted to present our study findings according to the Standards of Reporting of Diagnostic Accuracy (STARD) initiative guidelines. ${ }^{13}$ All but one of the 25 items on the STARD checklist have been satisfied. It was impractical for us to undertake BDR testing blind to the diagnosis of observed wheeze as recruitment and measurement were frequently undertaken by the same investigators.

This is the first study to describe the diagnostic characteristics of BDR testing in children with mild intermittent asthma, a group where diagnosis on reported symptoms is most challenging. ${ }^{14}$ The children who had the features of the "gold standard" for the disease were clearly defined. They had been observed to wheeze and were documented to have responded to treatment with a bronchodilator, were asymptomatic with no physical signs of wheeze at the time of testing, and had infrequent wheeze not meriting treatment with prophylactic asthma medication. These children with doctor-observed wheeze on a previous occasion are most likely to resemble those who present with reported symptoms but no physical signs in a primary care or outpatient setting. Children without the disease were those with no history of reported or observed wheeze- that is, no history of wheeze or difficulty in breathing.

We have shown that a positive BDR test is specific for recent wheeze but is fairly insensitive. In other words, a

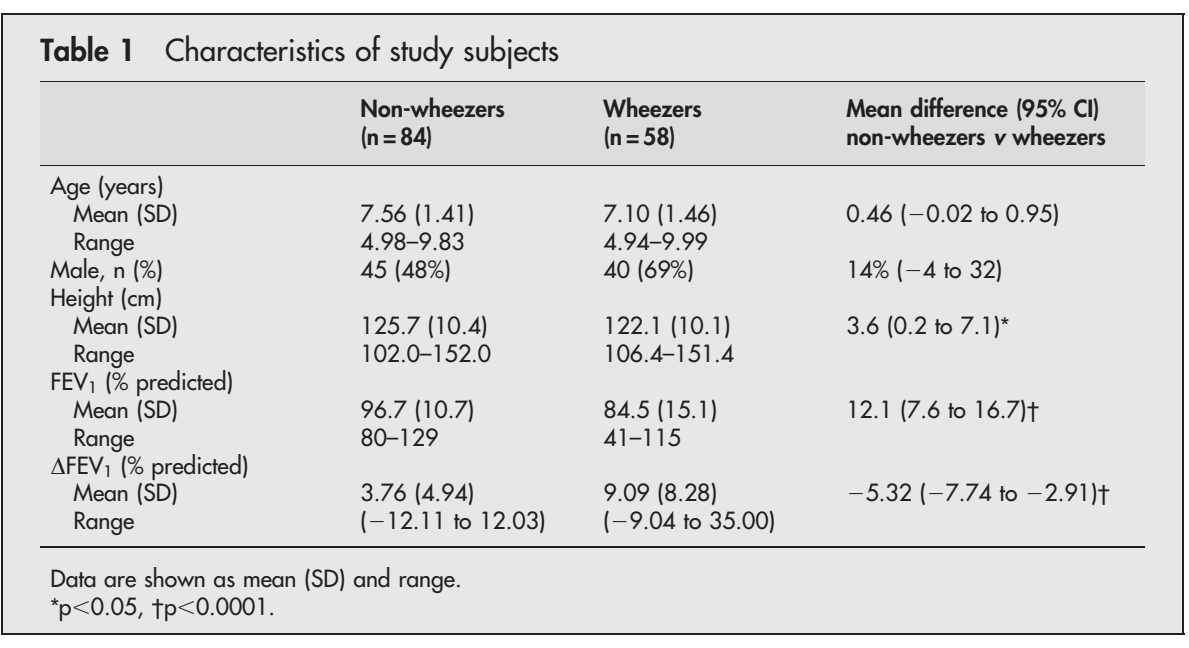


Table 2 Comparison of ROC cut off values for bronchodilator responsiveness (BDR)

\begin{tabular}{lllll}
\hline BDR & $\begin{array}{l}\text { Non-wheezers } \\
(\mathbf{n}=58)\end{array}$ & $\begin{array}{l}\text { Wheezers } \\
(\mathbf{n}=\mathbf{8 4})\end{array}$ & $\begin{array}{l}\text { Sensitivity } \\
\%(95 \% \mathrm{CI})\end{array}$ & $\begin{array}{l}\text { Specificity } \\
\%(95 \% \mathrm{Cl})\end{array}$ \\
\hline $6 \%$ & 56 & 34 & $59(46$ to 70$)$ & $66(55$ to 74$)$ \\
$9 \%$ & 72 & 29 & $50(38$ to 62$)$ & $86(78$ to 92$)$ \\
$12 \%$ & 82 & 20 & $35(24$ to 47$)$ & $98(92$ to 99$)$ \\
\hline
\end{tabular}

positive response is much more likely in children with wheeze than in children without wheeze, but about half of the wheezers will be missed. Positive and negative likelihood ratios of over 5 and under 0.2 , respectively, for a test are considered strongly diagnostic. ${ }^{15}$ We have calculated the positive and negative predictive values for positive and negative tests as clinicians can be misled by just the expression of the likelihood ratio. ${ }^{16}$ Although baseline $\mathrm{FEV}_{1}$ was reduced in the wheezing group, it was neither sensitive nor specific in identifying previous wheeze (data not shown).

Several groups have reported BDR response in healthy controls $^{5}{ }^{17-21}$ and asthmatic children, ${ }^{11}$ but the asthmatics included children who were on prophylactic treatment and therefore had more than mild intermittent asthma. These are more likely to be children where the diagnosis is not in doubt.

Values of $9 \%$ and $10.2 \%$ threshold change for BDR have been derived from upper $95 \%$ of response to bronchodilator and from repeatability studies in healthy populations respectively. ${ }^{5}$ This study has gone further in describing the test characteristics of BDR for patients where there is a diagnostic challenge for the clinician.

As well as the specificity and sensitivity, a measure of performance for a test using a ROC curve is given by the AUC. $^{22}$ A useless test would have an AUC of 0.50, the area being described by the line of identity. Generally, a test is considered discriminating if the AUC is more than $0.70 .{ }^{23}$ The AUC for BDR measurement in this study was 0.71, which suggests that BDR testing could be useful for identifying children with previous wheeze

Bronchial responsiveness to methacholine challenge may also be used to identify children who may have asthma. However, sensitivity and specificity for doctor-diagnosed asthma were $76 \%$ and $50 \%$, respectively, for a $15 \%$ fall in $\mathrm{FEV}_{1}$ at a dose of $1.8 \mathrm{mg} / \mathrm{ml}$ in a group of 6 year old children. ${ }^{24}$ The positive and negative likelihood ratios derived from these figures are approximately 1.5 (95\% CI 1.3 to 1.8 ) and 0.5 (95\% CI 0.3 to 0.7 ). At first sight it would appear that measurements of bronchial responsiveness do not perform as well as BDR testing in identifying children with asthma. This may be because the subjects with disease had doctordiagnosed asthma rather than doctor-observed wheeze. Children with doctor-diagnosed asthma are significantly different from those with doctor-observed wheeze with respect to atopic status and may include subjects with reported symptoms which are not caused by asthma. ${ }^{25}$

As the prevalence of asthma in the community is uncertain because of the inaccuracy of the reporting of symptoms, our data can be summarised by expressing our results over a range of hypothetical prevalences. As a child who presents

Table 3 Predictive values of a positive BDR of 9\%

\begin{tabular}{lll}
\hline $\begin{array}{l}\text { Pre-test probability } \\
\text { (estimated prevalence) }\end{array}$ & PPV & NPV \\
\hline $10 \%$ & $29 \%$ & $94 \%$ \\
$30 \%$ & $61 \%$ & $80 \%$ \\
$50 \%$ & $78 \%$ & $63 \%$ \\
\hline
\end{tabular}

PPV, positive predictive value; NPV, negative predictive value. with respiratory symptoms in primary care is more likely to have had wheeze than the general population, the prevalence of wheeze in the primary care population will be higher, and even higher in the group referred from general practice for a specialist opinion. Positive and negative predictive values can be calculated for these estimated prevalences. With increasing prevalence, the predictive values will change (table 3 ). How important knowledge of these values is will depend on the importance of the diagnosis when made. An improvement in the rate of diagnosis in the community from $10 \%$ to $29 \%$ may not be considered large enough to introduce BDR testing as a screening test as it would mean that, for every child with asthma, more than two children without the disease would be treated. However, where individuals are concerned enough to seek specialist advice, an increase from $50 \%$ pre-test probability to $78 \%$ post-test probability could be considered helpful.

In conclusion, we have described the diagnostic accuracy of BDR using spirometric testing in school age children. Although BDR should not be considered as a stand alone test, when combined with results from skin prick testing for determining allergic sensitisation, ${ }^{25}$ concordant results may increase confidence in diagnosing (or excluding the diagnosis of) asthma.

\section{ACKNOWLEDGEMENTS}

The authors thank Professor Michael Healy who gave statistical advice and approved the final manuscript.

\section{Authors' affiliations}

\section{Dundas, E Y Chan, P D Bridge, S A McKenzie, Department of}

Paediatric Respiratory Medicine, The Royal London Hospital, Barts and The London NHS Trust, London E1 1BB, UK

E Y Chan was supported by Asthma UK.

\section{REFERENCES}

1 National Institutes of Health. National asthma education program: executive summary: guidelines for the diagnosis and management of asthma. Bethesda, MA: National Institutes of Health, 1991.

2 British Thoracic Society/Scottish Intercollegiate Guideline Network. British guideline on the management of asthma. Thorax 2003;58(Suppl I):i1-94.

3 Borrill Z, Houghton C, Sullivan PJ, et al. Retrospective analysis of evidence base for tests used in diagnosis and monitoring of disease in respiratory medicine. BMJ 2003;327:1136-8.

4 Van Noord JA, Smeets J, Clement J, et al. Assessment of reversibility of airflow obstruction. Am J Respir Crit Care Med 1994;150:551-4.

5 Dales RE, Spitzer WO, Tousignant $P$, et al. Clinical interpretation of airway response to a bronchodilator. Epidemiologic considerations. Am Rev Respir Dis 1988; 138:317-20.

6 American Thoracic Society. Lung function testing: selection of reference values and interpretative strategies. Am Rev Respir Dis 1991;144:1202-18.

7 Strachan DP. Repeatability of ventilatory function measurements in a population survey of 7 year old children. Thorax 1989:44:474-9.

8 Polgar GP. Pulmonary function testing in children: techniques and standards, 1 st ed. New York: WB Saunders, 1971.

9 British Thoracic Society. Asthma in adults and schoolchildren. BTS guidelines on asthma management 1995. Thorax 1997;52(Suppl 1):S2-5, S20.

10 Bridge PD, McKenzie SA. Bronchodilator responsiveness testing in young children. Arch Dis Child 2001;84:525.

11 Waalkens HJ, Merkus PJ, Van Essen-Zandvliet EE, et al. Assessment of bronchodilator response in children with asthma. Dutch CNSLD Study Group. Eur Respir J 1993:6:645-51.

12 Habbema JDF, Eijkmans R, Krijnen $P$, et al. Analysis of data on the accuracy of diagnostic tests. In: Knottnerus JA, ed. The evidence base of clinical diagnosis. London: BMJ Books, 2002:117-43. 
13 Bossuyt PM Reitsma JB, Bruns DE, et al. Towards complete and accurate reporting of studies of diagnostic accuracy: the STARD initiative. BMJ 2003;326:41-4.

14 Cane RS, Ranganathan SC, McKenzie SA. What do parents of wheezy children understand by "wheeze"? Arch Dis Child 2000:82:327-32.

15 Jaeschke R, Guyatt G, Sackett DL. Users' guides to the medical literature. III. How to use an article about a diagnostic test. A. Are the results of the study valid? Evidence-Based Medicine Working Group. JAMA 1994;271:389-91.

16 Bachmann LM, Steurer J, ter Riet G. Simple presentation of test accuracy may lead to inflated disease probabilities. BMJ 2003;326:393.

17 Dalen G, Kjellman B. Assessment of lung function on healthy children using an electronic spirometer and an air-flowmeter before and after inhalation of an adrenergic receptor stimulant. Acta Paediatr Scand 1979;68:103-8.

18 Ekwo EE, Weinberger MM, Dusdieker LB, et al. Airways responses to inhaled isoproterenol in normal children. Am Rev Respir Dis 1983; 127:108-9.
19 Casan P, Roca J, Sanchis J. Spirometric response to a bronchodilator. Reference values for healthy children and adolescents. Bull Eur Physiopathol Respir 1983; 19:567-9.

20 Lis G, Haluszka J. Standards in evaluating the test of obstruction reversibility based on the flow-volume curves. Pneumonol Alergol Pol 1991:59:20-4.

21 Pardos MC, Fuertes Fernandez-Espinar J, et al. Cut-off point for a positive bronchodilation test. An Esp Pediatr 2002;57:5-1 1 .

22 Zweig MH, Campbell G. Receiver-operating characteristic (ROC) plots: a fundamental evaluation tool in clinical medicine. Clin Chem 1993:39:561-77.

23 Hanley JA, MCNeil BJ. The meaning and use of the area under a receiver operating characteristic (ROC) curve. Radiology 1982;143:29-36.

24 Joseph-Bowen J, de Klerk NH, Firth MJ, et al. Lung function, bronchial responsiveness, and asthma in a community cohort of 6-year-old children. Am J Respir Crit Care Med 2004;169:850-4.

25 Chan EY, Dundas I, Bridge PD, et al. Are doctor-diagnosed asthma and doctor-observed wheeze the same? Eur Respir J 2003;22:387s.

\section{LUNG ALERT}

SV40 as a causative agent in mesothelioma: a new twist to the story

$\Delta$ Lopez-Rios F, Illei PB, Rusch V, Ladanyi M. Evidence against a role for SV40 infection in human mesotheliomas and high risk of false-positive PCR results owing to presence of SV40 sequences in common laboratory plasmids. Lancet 2004:364:1157-66

T

he potential causal role of simian virus 40 (SV40) in tumourigenesis is the most contentious issue in mesothelioma research of recent years. SV40 is a potent tumour

virus and can induce mesothelioma in experimental animals. However, clinical evidence linking SV40 with mesothelioma remains inconclusive. SV40 has been reported in human mesothelioma tissues in many, but not all, previous publications. Although the criteria for causality have not been satisfied, strategies targeting SV40 are already being pursued in clinical trials for mesothelioma.

Lopez-Rios et al used several lines of approach to show that PCR primers used to detect SV40 in many other studies were targeting sequences within the SV40 genome that are also present in common laboratory plasmids. "Positive" SV40 detection in the mesothelioma samples was actually amplication of the DNA of laboratory plasmids rather than that of SV40. Using carefully designed primers to avoid false positives from plasmid contamination, they confirmed that DNA of the SV40 T antigen was not present in 71 mesothelioma samples. To further prove the point, they showed that neither the RNA (by RT-PCR) nor protein (by immunohistochemistry) of SV40 T antigen were detectable in any of the 71 samples. The data strongly suggest that previous reports of SV40 in mesothelioma were the result of laboratory contamination rather than genuine viral infection of the human tissue.

The methodology of this paper appears robust and its results seriously question the evidence upon which proponents of SV40 base the causality theory. There is little doubt that it will spark a new round of debate on SV40 and mesothelioma.

Y C G Lee

Wellcome Advanced Fellow, University College London and Honorary Chest Physician, Osler Chest Unit, Oxford, UK; ycgarylee@hotmail.com 